\title{
COMPACT ABELIAN PRIME ACTIONS ON VON NEUMANN ALGEBRAS
}

\author{
KLAUS THOMSEN
}

\begin{abstract}
We classify the compact abelian actions on semifinite injective von Neumann algebras with factor fixed point algebra. The method uses that the (nonzero) eigenspaces of such an action contain unitaries which give rise to a classifying invariant.
\end{abstract}

\section{INTRODUCTION}

Jones and Takesaki have used Ocneanu's results on discrete amenable group actions on von Neumann algebras to classify compact abelian group actions on semifinite injective von Neumann algebras [4, 5]. The basic idea is to use Ocneanu's results on the dual action and then use Takesaki duality to classify up to stable conjugacy. Finally, they introduce the inner invariant, see [5], to classify up to conjugacy. The main result of [5] handles the general case which is severely complicated when the crossed product is not a factor.

The purpose of the present paper is to present another approach which works in a more restrictive setting, namely in the setting of prime actions, i.e. actions with a factor as fixed point algebra. By a result of Paschke, [10], this condition is fulfilled if and only if the crossed product is a factor. Our starting observation was that for a compact abelian prime action every nonzero eigenspace contains a unitary eigenoperator unless the algebra is of type III and the fixed point algebra is of type $\mathrm{II}_{1}$, see $\S 2$. By excluding this possibility the existence of unitary eigenoperators translates the problem of classifying compact abelian prime actions into the problem of classifying cocycle crossed actions of the dual group on the fixed point algebra. This translation is done in $\S 3$ and we introduce an invariant for cocycle crossed actions which generalizes the characteristic invariant for ordinary discrete actions. We then investigate the relationship between the algebra and the fixed point algebra in $\S 4$. In $\S 5$ we prove uniqueness, up to conjugacy, for actions on the hyperfinite type II factors, subject to the condition that the fixed point algebra has trivial relative commutant. In $\S 6$ we solve the problem by using the results of Ocneanu from [8], together with the results obtained in the previous sections. In $\S 7$ it is shown how our approach gives a classification result for certain locally compact abelian actions, namely

Received by the editors March 15, 1988.

1980 Mathematics Subject Classification (1985 Revision). Primary 46L55; Secondary 46L40. 
the almost periodic prime actions. Finally, we give some supplementary results in $\S 8$ which can be derived from our methods and give a little information on actions on type III algebras.

To describe our main result, let $\alpha: G \rightarrow$ Aut $\mathscr{M}$ and $\beta: G \rightarrow$ Aut $\mathscr{N}$ be two prime actions of the compact abelian second countable group $G$ on the semifinite injective von Neumann algebras $\mathscr{M}$ and $\mathscr{N}$. We introduce the invariant $(N(\alpha), \Omega(\alpha))$ with the aid of the unitary eigenoperators. Here $N(\alpha)$ is a subgroup of $\widehat{G}$ and $\Omega(\alpha): \widehat{G} \times N(\alpha) \rightarrow T$ is a bihomomorphism which is antisymmetric on $N(\alpha) \times N(\alpha)$. Not surprisingly it turns out that $(N, \Omega)$ is the characteristic invariant for the dual action $\hat{\alpha}$, see [5]. We find that $\alpha$ and $\beta$ are conjugate if and only if the fixed point algebras $\mathscr{M}^{\alpha}$ and $\mathscr{N}^{\beta}$ are *isomorphic, $N(\alpha)=N(\beta)$ and $\Omega(\alpha)=\Omega(\beta)$. This is a direct generalization of Jones' result for prime actions on $R$, [4], but is obtained by completely different means. To compare with the methods of [5], observe that [5] gives the following result: $\alpha$ and $\beta$ are conjugate if and only if $\mathscr{M} \times{ }_{\alpha} G$ and $\mathscr{N} \times{ }_{\beta} G$ are *-isomorphic, the characteristic invariants of $\hat{\alpha}$ and $\hat{\beta}$ are the same and $\alpha$ and $\beta$ have the same inner invariant. Thus our result removes the inner invariant and exchanges $*$-isomorphism of the crossed products with $*$-isomorphism of the fixed point algebras.

It should be noted that the main difficulty in [5] is precisely that the crossed product need not be a factor and that the method used in [5] is much more direct in case of prime actions, thanks to the theorem of Paschke. However, we feel that the elimination of the inner invariant, which is hard to understand, is an advantage.

Throughout the paper we let $G$ denote a compact abelian group and consider only faithful actions of $G$, except when the contrary is explicitly stated.

Finally, I will like to thank Uffe Haagerup for pointing out that not all prime $G$-actions have full unitary spectrum and Niels Munch for many helpful discussions.

\section{THE EXISTENCE OF UNITARY EIGENOPERATORS}

Definition 2.1. An action $\alpha: G \rightarrow$ Aut $\mathscr{M}$ has full unitary spectrum if every nonzero eigenspace contains a unitary.

We first show that for prime actions the Arveson spectrum is a subgroup of $\widehat{G}$ and, because we have adopted the convention that all actions are faithful, it follows that $\operatorname{Sp}(\alpha)=\widehat{G}$ for prime actions. This is presumably well known.

Lemma 2.2. Let $\alpha: G \rightarrow$ Aut $\mathscr{M}$ be a prime action. Then $\operatorname{Sp}(\alpha)=\widehat{G}$.

Proof. For $p \in \widehat{G}$, let $\mathscr{M}(p)=\left\{a \in \mathscr{M} \mid \alpha_{g}(a)=\langle g, p\rangle a, g \in G\right\}$ be the corresponding eigenspace. Assume $\mathscr{M}(p) \neq 0$ and $\mathscr{M}(q) \neq 0$. If $\mathscr{M}(p) \mathscr{M}(q)=$ 0 , we have $\mathscr{M}(p) * \mathscr{M}(p) \mathscr{M}(q) \mathscr{M}(q) *=0$, which is impossible since $\mathscr{M}(p) *$ $\mathscr{M}(p)$ and $\mathscr{M}(q) \mathscr{M}(q)^{*}$ are both nonzero two-sided ideals in the factor $\mathscr{M}^{\alpha}$. Hence $0 \neq \mathscr{M}(p) \mathscr{M}(q) \subseteq \mathscr{M}(p+q)$, showing that $\operatorname{Sp}(\alpha)$ is a group. Since $\alpha$ is faithful, $\operatorname{Sp}(\alpha)=\widehat{G}$. 
It follows that prime $G$-actions have full unitary spectrum if and only if all eigenspaces contain a unitary. The main result of this section is the following

Theorem 2.3. Let $\alpha: G \rightarrow$ Aut $\mathscr{M}$ be a prime action. If $\alpha$ does not have full unitary spectrum, then $\mathscr{M}$ is of type $\mathrm{III}$ and $\mathscr{M}^{\alpha}$ is of type $\mathrm{II}_{1}$.

The proof will be based on the two following lemmas.

Lemma 2.4. If $\alpha: G \rightarrow$ Aut $\mathscr{M}$ is a prime action, then every eigenspace contains an isometry or a coisometry.

Proof. Fix $p \in \widehat{G}$ and let $\left\{v_{i}\right\}_{i \in I}$ be a maximal family of partial isometries in $\mathscr{M}(p)$ such that $v_{p}^{*} v_{q}=v_{p} v_{q}^{*}=0$ for $p \neq q$. Set $v=\sum_{i \in I} v_{i}, e=v v^{*}$ and $f=v^{*} v$. Then $v \in \mathscr{M}(p), e, f \in \mathscr{M}^{\alpha}$. We assert that $(\mathbf{I}-e) \mathscr{M}(p)(\mathbf{I}-f)=0$. Indeed if $m \in(\mathbf{I}-e) \mathscr{M}(p)(\mathbf{I}-f)$ is nonzero, the polardecomposition of $m$ produces a nonzero partial isometry $w$ in $\mathscr{M}(p)$ such that $w w^{*} \leq \mathbf{I}-e$ and $w^{*} w \leq \mathbf{I}-f$. This contradicts the maximality of $\left\{v_{i}\right\}_{i \in I}$. Therefore the assertion holds and we get furthermore that

$$
u_{1}(\mathbf{I}-e) u_{1}^{*} \mathscr{M}(p) u_{2}(\mathbf{I}-f) u_{2}^{*}=0
$$

for all unitaries $u_{1}, u_{2}$ in $\mathscr{M}^{\alpha}$. Thus $z(\mathbf{I}-e) \mathscr{M}(p) z(\mathbf{I}-f)=0$, where $z(\cdot)$ denotes the central support in $\mathscr{M}^{\alpha}$. Since $\mathscr{M}^{\alpha}$ is a factor and $\mathscr{M}(p) \neq 0$ by Lemma 2.2, we conclude that $e=\mathbf{I}$ or $f=\mathbf{I}$.

Lemma 2.5. Let $\alpha: G \rightarrow$ Aut $\mathscr{M}$ be a prime action and assume that $\mathscr{M}^{\alpha}$ is properly infinite. Then $\alpha$ has full unitary spectrum.

Proof. Fix $p \in \widehat{G}$. By Lemma 2.4 we can assume that $\mathscr{M}(p)$ contains an isometry $v$ (otherwise consider $\left.\mathscr{M}(-p)=\mathscr{M}(p)^{*}\right)$. Assume first that $\mathscr{M}^{\alpha}$ is of type III. Then $\mathscr{M}^{\alpha}$ contains an isometry $w$ such that $w w^{*}=v v^{*}$. Then $w^{*} v$ is a unitary in $\mathscr{M}(p)$. Assume next that $\mathscr{M}^{\alpha}$ is semifinite. Then $\mathscr{M}^{\alpha}=$ $\mathscr{N} \otimes \mathscr{B}$, where $\mathscr{N}$ is finite and $\mathscr{B}$ is type $\mathrm{I}_{\infty},[14$, Chapter V, Proposition 1.40]. Then $\mathscr{M}=\mathscr{M}_{1} \otimes \mathscr{B}$ where $\mathscr{M}_{1}=\mathscr{B}^{\prime} \cap \mathscr{M}$ and $\alpha=\left.\alpha\right|_{\mathscr{M}_{1}} \otimes$ id. Since $\mathscr{M}(p)=\mathscr{M}(p) \cap \mathscr{M}_{1} \otimes \mathscr{B}$, we can assume that $v$ is an isometry in $\mathscr{M}(p) \cap \mathscr{M}_{1}$. Then $v v^{*} \otimes \mathbf{I}$ is an infinite projection in $\mathscr{M}^{\alpha}$, so there is an isometry $w \in \mathscr{M}^{\alpha}$ such that $w w^{*}=v v^{*} \otimes \mathbf{I}$. Then $w^{*}(v \otimes \mathbf{I})$ is the desired unitary in $\mathscr{M}(p)$.

Proof of Theorem 2.3. Assume $\mathscr{M}(p)$ contains an isometry $v$ but no unitary for some $p \in \widehat{G}$. By Lemma $2.5 \mathscr{M}^{\alpha}$ must be finite. If $\operatorname{dim} \mathscr{M}^{\alpha}<\infty$, $\mathscr{M}=\mathscr{M} \cap\left(\mathscr{M}^{\alpha}\right)^{\prime} \otimes \mathscr{M}^{\alpha}$, so $\left.\alpha\right|_{\mathscr{M} \cap\left(\mathscr{M}^{\alpha}\right)^{\prime}}$ is an ergodic action. But ergodic actions always have full unitary spectrum, see [9], so this rules out the possibility that $\operatorname{dim} \mathscr{M}^{\alpha}<\infty$. Hence $\mathscr{M}^{\alpha}$ is of type II ${ }_{1}$. Let $H=\{z p \mid z \in \mathbf{Z}\}$. If $k \in \mathbf{N}, v^{k}$ is an isometry in $\mathscr{M}(k p)$ and $\mathscr{M}(k p)=\mathscr{M}^{\alpha} v^{k}$. In the same way, $\mathscr{M}(-k p)=$ $\left(v^{*}\right)^{k} \mathscr{M}^{\alpha}$. It follows that the von Neumann algebra $\mathscr{N}$ generated by $v$ and $\mathscr{M}^{\alpha}$ is the same as the one generated by $\bigcup_{h \in H} \mathscr{M}(h)$. Hence $\int_{H^{\perp}} \alpha_{g}(\cdot) d g$ defines a normal faithful conditional expectation from $\mathscr{M}$ onto $\mathscr{N}$. By [13, 
$\S \S 2$ and 3], $\mathscr{N}$ is a type III factor. By [14, Chapter V, Lemma 2.29], $\mathscr{M}$ is of type III also.

There is a modular automorphism group of the hyperfinite $\mathrm{III}_{\lambda}$-factor, $0<$ $\lambda<1$, which is periodic so that it corresponds to an action of $\mathbf{T}$, and satisfies that the fixed point algebra is a $\mathrm{II}_{1}$-factor and is the only eigenspace containing a unitary, see [13]. So Theorem 2.3 is the best we can expect concerning the existence of unitary eigenoperators for general prime $G$-actions. However, we have the following

Proposition 2.6. Let $\alpha: G \rightarrow$ Aut $\mathscr{M}$ be a prime action. If $G$ is totally disconnected, $\alpha$ has full unitary spectrum.

Proof. By Theorem 2.3 we can assume that $\mathscr{M}^{\alpha}$ is a II ${ }_{1}$-factor. If $v$ is an isometry in $\mathscr{M}(p)$, then $v^{k} \in \mathscr{M}(k p), k \in \mathbf{N}$. By assumption $\widehat{G}$ is a torsion group so $v^{k} \in \mathscr{M}^{\alpha}$ for some $k \in \mathbf{N}$. Thus $v^{k}$ is an isometry in a finite factor, i.e. $v^{k}$ is unitary and so is $v$.

\section{THE INVARIANT FOR PRIME ACTIONS WITH FULL UNITARY SPECTRUM}

Let $\alpha: G \rightarrow$ Aut $\mathscr{M}$ be a prime action. By Theorem 2.3 we know that it not too restrictive to assume that $\alpha$ has full unitary spectrum. Doing so, we can find unitaries $\left\{V_{p} \mid p \in \widehat{G}\right\}$ in $\mathscr{M}$ such that

$$
V_{p} \in \mathscr{M}(p), \quad p \in \widehat{G} .
$$

Observe that $\mathscr{M} \cap\left(\mathscr{M}^{\alpha}\right)^{\prime}$ reduces $\alpha$ and that $\left.\alpha\right|_{\mathscr{M} \cap\left(\mathscr{M}^{\alpha}\right)^{\prime}}$ is an ergodic, but not necessarily faithful action. Define $N(\alpha)=\operatorname{Sp}\left(\left.\alpha\right|_{\mathscr{M} \cap\left(\mathscr{M}^{\alpha}\right)^{\prime}}\right)$. Then $p \in N(\alpha)$ if and only if $\mathscr{M}(p) \cap\left(\mathscr{M}^{\alpha}\right)^{\prime} \neq\{0\}$, and for such a $p$, we can find a unitary in $\mathscr{M}(p) \cap\left(\mathscr{M}^{\alpha}\right)^{\prime}$ because $\alpha$ is ergodic on $\mathscr{M} \cap\left(\mathscr{M}^{\alpha}\right)^{\prime}$. Thus we can choose the unitaries $V_{p}$ such that

$$
V_{p} \in \mathscr{M}(p) \cap\left(\mathscr{M}^{\alpha}\right)^{\prime}, \quad p \in N(\alpha) .
$$

Observe that $N(\alpha)$ is a subgroup of $\widehat{G}$ and that the $V_{p}$ 's normalize $\mathscr{M}^{\alpha}$ and $\mathscr{M} \cap\left(\mathscr{M}^{\alpha}\right)^{\prime}$. It follows from the ergodicity of $\alpha$ on $\mathscr{M} \cap\left(\mathscr{M}^{\alpha}\right)^{\prime}$ that we can define a function

$$
\Omega(\alpha): \widehat{G} \times N(\alpha) \rightarrow \mathbf{T}
$$

by

$$
V_{p} V_{q} V_{p}^{*}=\Omega(\alpha)(p, q) V_{q}, \quad p \in \widehat{G}, q \in N(\alpha) .
$$

It is straightforward to check that another choice of unitaries satisfying (a) and (b) yields the same function $\Omega(\alpha)$. In particular, we see from this that the pair $(\Omega(\alpha), N(\alpha))$ only depends on the conjugacy class of $\alpha$.

Lemma 3.1. $\Omega(\alpha)$ is a bihomomorphism which is antisymmetric on $N(\alpha) \times$ $N(\alpha)$, i.e. we have

(i) $\Omega(\alpha)\left(p_{1}+p_{2}, q_{1}\right)=\Omega(\alpha)\left(p_{1}, q_{1}\right) \Omega(\alpha)\left(p_{2}, q_{1}\right)$, 
(ii) $\Omega(\alpha)\left(p_{1}, q_{1}+q_{2}\right)=\Omega(\alpha)\left(p_{1}, q_{1}\right) \Omega(\alpha)\left(p_{1}, q_{2}\right)$, and

(iii) $\Omega(\alpha)\left(q_{1}, q_{1}\right)=1$ for all $p_{1}, p_{2} \in \widehat{G}, q_{1}, q_{2} \in N(\alpha)$.

Proof. (iii) is obvious. (i) and (ii) follow by using that $V_{p_{1}+p_{2}} V_{p_{1}}^{*} V_{p_{2}}^{*} \in \mathscr{M}^{\alpha}$, $p_{1}, p_{2} \in \widehat{G}$, and that $V_{q_{1}+q_{2}} V_{q_{1}}^{*} V_{q_{2}}^{*} \in \mathbf{C}, q_{1}, q_{2} \in N(\alpha)$. We leave the details to the reader.

With the following definition we follow the terminology from [8].

Definition 3.2. Let $H$ be a discrete abelian group and $\mathscr{M}$ a von Neumann algebra. A cocycle crossed action of $H$ on $\mathscr{M}$ is a pair $(\alpha, U)$, where $\alpha: H \rightarrow$ Aut $\mathscr{M}$ and $U: H \times H \rightarrow U(\mathscr{M})$ satisfy

(i) $\alpha_{g} \alpha_{h}=\operatorname{Ad} U_{g, h} \alpha_{g+h}$,

(ii) $U_{g, h} U_{g+h, k}=\alpha_{g}\left(U_{h, k}\right) U_{g, h+k}$,

(iii) $U_{0, g}=U_{g, 0}=\mathbf{I}, g, h, k \in H$.

Let $(\alpha, U)$ be a cocycle crossed action of $H$ on $\mathscr{M}$, and assume that $\mathscr{M}$ acts on the Hilbertspace $\mathscr{H}$. Following Sutherland, [11], we define the operators $I(x), x \in \mathscr{M}$, and $\pi(g), g \in H$, on $L^{2}(H, \mathscr{H})$ by

$$
\begin{gathered}
(I(x) \psi)(h)=\alpha_{-h}(x) \psi(h), \\
(\pi(g) \psi)(h)=U_{-h, g} \psi(h-g), \quad \psi \in L^{2}(H, \mathscr{H}) .
\end{gathered}
$$

One finds the following relations:

$$
\pi(g) I(x) \pi(g)^{*}=I\left(\alpha_{g}(x)\right)
$$

and

$$
\pi(g) \pi(h) \pi(g+h)^{*}=I\left(U_{g, h}\right), \quad x \in \mathscr{M}, g, h \in H .
$$

Definition 3.3. The crossed product $\mathscr{M} \times_{\alpha, U} H$ is defined to be the von Neumann algebra generated by the operators $I(x), x \in \mathscr{M}$, and $\pi(g), g \in H$.

In the terminology of [11], $\mathscr{M} \times{ }_{\alpha, U} H$ is a regular extension of $\mathscr{M}$ by $H$. As for crossed products by ordinary actions of abelian groups, we have an action $\hat{\alpha}: \widehat{H} \rightarrow$ Aut $\mathscr{M} \times_{\alpha, U} H$ such that $\hat{\alpha}_{p}(\pi(g))=\langle g, p\rangle \pi(g), g \in H, p \in \widehat{H}$, and $I(\mathscr{M})$ is the fixed point algebra of $\hat{\alpha},($ see $[11$, p. 150]).

Proposition 3.4. Let $\beta: G \rightarrow$ Aut $\mathscr{M}$ be an action with full unitary spectrum and choose unitaries $V_{p} \in \mathscr{M}(p), p \in \widehat{G}$, such that $V_{0}=\mathbf{I}$. Define $(\alpha, U)$ by $\alpha_{p}=\operatorname{Ad} V_{p \mid \mathscr{M}^{\beta}}$ and $U_{p, q}=V_{p} V_{q} V_{p+q}^{*}, p, q \in \widehat{G}$. Then $(\alpha, U)$ is a cocycle crossed action and $\hat{\alpha}$ is conjugate to $\beta$ if $\mathscr{M}$ is $\sigma$-finite.

Proof. That $(\alpha, U)$ is a cocycle crossed action follows from straightforward calculations. Observe that $\mathscr{N}=\operatorname{span}\left\{I(x) \pi(g) \mid x \in \mathscr{M}^{\beta}, g \in \widehat{G}\right\}$ is a weakly dense *-subalgebra of $\mathscr{M}^{\beta} \times_{\alpha, U} \widehat{G}$, that $P_{\hat{\alpha}}=\int_{G} \hat{\alpha}_{p}(\cdot) d p$ defines a faithful normal conditional expectation from $\mathscr{M}^{\beta} \times_{\alpha, U} \widehat{G}$ onto $I(\mathscr{M})$ and that every element $m$ in $\mathscr{N}$ admits a unique decomposition $m=\sum_{p \in \widehat{G}} I\left(x_{p}\right) \pi(p)$, where 
$x_{p} \in \mathscr{M}^{\beta}, p \in \widehat{G}$. Thus we can define an injective $*$-homomorphism $\psi: \mathscr{N} \rightarrow$ by

$$
\psi\left(\sum_{p \in \widehat{G}} I\left(x_{p}\right) \pi(p)\right)=\sum_{p \in \widehat{G}} x_{p} V_{p} .
$$

We claim that $\psi$ extends to a $*$-isomorphism of $\mathscr{M}^{\beta} \times_{\alpha, U} \widehat{G}$ onto $\mathscr{M}$ conjugating $\hat{\alpha}$ and $\beta$. This is an easy consequence of the next lemma which we shall also need later.

Lemma 3.5. Let $\mathscr{M}_{i}, \quad i=1,2$, be von Neumann algebras with weakly dense *-subalgebras $\mathscr{M}_{i}^{0} \subseteq \mathscr{M}_{i}, i=1,2$, and von Neumann subalgebras $\mathscr{N}_{i} \subseteq \mathscr{M}_{i}^{0} \subseteq$ $\mathscr{M}_{i}, \quad i=1,2$. Assume that there are faithful normal conditional expectations $P_{i}: \mathscr{M}_{i} \rightarrow \mathscr{N}_{i}, \quad i=1,2$, and a bijective *-homomorphism $\psi: \mathscr{M}_{1}^{0} \rightarrow \mathscr{M}_{2}^{0}$ such that $P_{2} \circ \psi=\psi \circ P_{1}$. If $\mathscr{N}_{2}$ is $\sigma$-finite, there is a unique $*$-isomorphism $\bar{\psi}: \mathscr{M}_{1} \rightarrow$ $\mathscr{M}_{2}$ extending $\psi$.

Proof. Let $\omega$ be a normal faithful state on $\mathscr{N}_{2}$, which exists because $\mathscr{N}_{2}$ is $\sigma$-finite. Then $\tilde{\omega}=\omega \circ P_{2}$ is a normal faithful state on $\mathscr{M}_{2}$. Observe that $\psi$ restricts to a $*$-isomorphism from $\mathscr{N}_{1}$ onto $\mathscr{N}_{2}$. So when we define $\mu=$ $\tilde{\omega} \circ \psi \circ P_{1}$ on $\mathscr{M}_{1}$, we obtain a normal faithful state. Note that $\tilde{\omega} \circ \psi=\omega \circ P_{2} \circ \psi=$ $\omega \circ \psi \circ P_{1}=\mu$ on $\mathscr{M}_{1}^{0}$. It is now standard to use the GNS-representations of $\omega$ and $\mu$ to extend $\psi$.

To apply Proposition 3.4, Lemma 3.5 and results from other papers, we now assume that all von Neumann algebras we consider have separable predual.

Definition 3.6. Let $(\alpha, U)$ and $(\beta, V)$ be cocycle crossed actions of $H$ on $\mathscr{M}$ and $\mathcal{N}$, respectively. Then $(\alpha, U)$ and $(\beta, V)$ are called cocycle conjugate if there is a *-isomorphism $\theta: \mathscr{M} \rightarrow \mathscr{N}$ and a map $W: H \rightarrow U(\mathscr{M})$ such that

$$
W_{g} \alpha_{g}\left(W_{h}\right)=\theta^{-1}\left(V_{g, h}\right) W_{g+h} U_{g, h}^{*}
$$

$$
\beta_{g}=\theta \operatorname{Ad} W_{g} \alpha_{g} \theta^{-1}, \quad g, h \in H
$$

The importance of this notion, which generalizes the usual notion for ordinary actions, lies in the following result.

Lemma 3.7. $(\alpha, U)$ and $(\beta, V)$ are cocycle conjugate if and only if $\hat{\alpha}$ and $\hat{\beta}$ are conjugate.

Proof. Let first $\theta$ and $W$ be such that (a) and (b) of Definition 3.6 are satisfied. Let $I^{\alpha}(x), x \in \mathscr{M}, I^{\beta}(x), x \in \mathscr{N}$, and $\pi^{\alpha}(g), \pi^{\beta}(g), g \in H$, be the 
operators defined above such that $\mathscr{M} \times_{\alpha, U} H$ is generated by $I^{\alpha}(x), x \in \mathscr{M}$, and $\pi^{\alpha}(g), g \in H$ and $\mathcal{N} \times{ }_{\beta, V} H$ is generated by $I^{\beta}(x), x \in \mathscr{N}$ and $\pi^{\beta}(g)$, $g \in H$. We can then define a bijective $*$-homomorphism $\psi$ between weakly dense *-subalgebras by

$$
\psi\left(\sum_{g \in H} I^{\alpha}\left(x_{g}\right) \pi^{\alpha}(g)\right)=\sum_{g \in H} I^{\beta}\left(\theta\left(x_{g} W_{g}^{*}\right)\right) \pi^{\beta}(g) .
$$

By Lemma $3.5 \psi$ extends to a *-isomorphism $\bar{\psi}: \mathscr{M} \times_{\alpha, U} H \rightarrow \mathscr{N} \times_{\beta, V} H$ which clearly satisfies $\bar{\psi} \hat{\alpha}_{p}=\hat{\beta}_{p} \bar{\psi}, p \in \widehat{H}$.

Conversely, assume that $\psi: \mathscr{M} \times{ }_{\alpha, U} H \rightarrow \mathscr{N} \times_{\beta, V} H$ is a $*$-isomorphism such that $\hat{\beta} \psi=\psi \hat{\alpha}$. It follows that $\psi$ restricts to a $*$-isomorphism mapping $I^{\alpha}(\mathscr{M})$ onto $I^{\beta}(\mathscr{N})$. Define $\theta: \mathscr{M} \rightarrow \mathscr{N}$ by $I^{\beta}(\theta(x))=\psi\left(I^{\alpha}(x)\right), x \in \mathscr{M}$. Since $\psi^{-1}\left(\pi^{\beta}(g)\right) \pi^{\alpha}(g)^{*} \in I^{\alpha}(\mathscr{M})$, we can define $W: H \rightarrow U(\mathscr{M})$ by $I^{\alpha}\left(W_{g}\right)=$ $\psi^{-1}\left(\pi^{\beta}(g)\right) \pi^{\alpha}(g)^{*}, g \in H$. We leave to the reader to check that $\theta$ and $W$ satisfy conditions (a) and (b) of Definition 3.6.

Finally, we need to identify an invariant for cocycle crossed actions on factors which corresponds to the invariant $(\Omega, N)$ introduced above. Let $(\alpha, U)$ be a cocycle crossed action of $H$ on the factor $\mathscr{M}$. Define $N(\alpha)=\left\{h \in H \mid \alpha_{h}\right.$ is inner $\}$, and define a map $\Omega(\alpha, U): H \times N(\alpha) \rightarrow \mathrm{T}$ by

$$
\Omega(\alpha, U)(g, h)=U_{g, h} \alpha_{h+g}\left(S_{h}^{*}\right) U_{h, g}^{*} S_{h}, \quad g \in H, h \in N(\alpha),
$$

where $S_{h} \in \mathscr{U}(\mathscr{M})$ are unitaries implementing $\alpha_{h}, h \in N(\alpha)$. Since $\mathscr{M}$ is a factor each $S_{h}$ is determined up to multiplication by a scalar, so $\Omega(\alpha, U)$ is clearly independent of the choice of the $S_{h}$ 's. It may not be obvious at a glance that $\Omega(\alpha, U)$ takes values in $\mathbf{T}$, but we prove the following lemma which implies this and other properties of $\Omega(\alpha, U)$, cf. Lemma 3.1.

Lemma 3.8. $N(\alpha)=N(\hat{\alpha})$ and $\Omega(\alpha, U)=\Omega(\hat{\alpha})$.

Proof. Let $\mathscr{M} \times{ }_{\alpha, U} H$ be generated by $I(x), x \in \mathscr{M}$, and $\pi(g), g \in H$. Assume $h \in N(\alpha)$ and choose $S_{h} \in \mathscr{U}(\mathscr{M})$ implementing $\alpha_{h}$. Then $\pi(h) I\left(S_{h}^{*}\right) \in$ $I(\mathscr{M})^{\prime}$ and $\hat{\alpha}_{p}\left(\pi(h) I\left(S_{h}^{*}\right)\right)=\langle h, p\rangle \pi(h) I\left(S_{h}^{*}\right), p \in \widehat{H}$. Hence $h \in N(\hat{\alpha})$. Conversely, if $h \in N(\hat{\alpha})$, there is a unitary $V_{h} \in \mathscr{M} \times_{\alpha, U} H \cap I(\mathscr{M})^{\prime}$ such that $\hat{\alpha}_{p}\left(V_{h}\right)=\langle h, p\rangle V_{h}, p \in \hat{H}$. Then $V_{h}^{*} \pi(h) \in I(\mathscr{M})$ and $I\left(\alpha_{h}(\cdot)\right)=$ $V_{h}^{*} \pi(h) I(\cdot) \pi(h)^{*} V_{h}$. Thus $\alpha_{h}$ is inner and $h \in N(\alpha)$. Hence $N(\alpha)=N(\hat{\alpha})$. Choose unitaries $V_{h} \in \mathscr{M} \times_{\alpha, U} H, h \in H$, such that $\hat{\alpha}_{p}\left(V_{h}\right)=\langle h, p\rangle V_{h}$, $p \in \hat{H}$, and $V_{h} \in \mathscr{M} \times_{\alpha, U} H \cap I(\mathscr{M})^{\prime}$ when $h \in N(\alpha)=N(\hat{\alpha})$. Then $V_{g} \in I(\mathscr{U}(\mathscr{M})) \pi(g), g \in H$, and $S_{h}=I^{-1}\left(V_{h}^{*} \pi(h)\right)$ implements $\alpha_{h}$ when 
$h \in N(\alpha)$. Thus we find, with $g \in H, h \in N(\alpha)$,

$$
\begin{aligned}
V_{g} V_{h} V_{g}^{*} V_{h}^{*} & =\pi(g) V_{h} \pi(g)^{*} V_{h}^{*} \\
& =\pi(g) \pi(h) \pi(g)^{*} \pi(g) \pi(h)^{*} V_{h} \pi(g)^{*} V_{h}^{*} \pi(h) \pi(h)^{*} \\
& =\pi(g) \pi(h) \pi(g)^{*} I\left(\alpha_{g}\left(S_{h}^{*}\right) S_{h}\right) \pi(h)^{*} \\
& =\pi(g) \pi(h) \pi(g)^{*} \pi(h)^{*} I\left(\alpha_{h} \alpha_{g}\left(S_{h}^{*}\right) \alpha_{h}\left(S_{h}\right)\right) \\
& =I\left(U_{g, h} U_{h, g}^{*}\right) I\left(U_{h, g} \alpha_{h+g}\left(S_{h}^{*}\right) U_{h, g}^{*} S_{h}\right) \\
& =I\left(U_{g, h} \alpha_{h+g}\left(S_{h}^{*}\right) U_{h, g}^{*} S_{h}\right) .
\end{aligned}
$$

Hence $\Omega(\alpha, U)=\Omega(\hat{\alpha})$ as .asserted.

Observe that by Lemma 3.7 the cocycle crossed action $(\alpha, U)$ constructed from $\beta$ in Proposition 3.4 is determined by $\beta$ up to cocycle conjugacy. Such a cocycle crossed action will be called a predual cocycle crossed action for $\beta$.

\section{The Relation Between $\mathscr{M}$ AND $\mathscr{M}^{\alpha}$}

Definition 4.1. An action $\alpha: g \rightarrow$ Aut $\mathscr{M}$ is called a dual action if there is a homomorphism $W: \widehat{G} \rightarrow \mathscr{U}(\mathscr{M})$ such that $\alpha_{g}\left(W_{p}\right)=\langle p, g\rangle W_{p}, g \in G$, $p \in \widehat{G}$.

The reason for this name is of course that by Landstad's characterization of abelian crossed products, [7], the condition of the definition implies that $\alpha$ is conjugate to the dual action on the crossed product $\widehat{G} \times{ }_{\text {ad } w} \mathscr{M}^{\alpha}$. We shall need the following alternative characterization of dual actions for later reference.

Lemma 4.2. Let $\alpha: G \rightarrow$ Aut $\mathscr{M}$ be an action with full unitary spectrum and choose a predual cocycle crossed action $(\check{\alpha}, U)$ for $\alpha$. Then $\alpha$ is a dual action if and only if there is a map $V: \widehat{G} \rightarrow \mathscr{U}\left(\mathscr{M}^{\alpha}\right)$ such that $U_{p, q}=\check{\alpha}_{p}\left(V_{q}^{*}\right) V_{p}^{*} V_{p+q}$, $p, q \in \widehat{G}$.

Proof. Assume first that $\alpha$ is a dual action and let $W$ be a homomorphism as in Definition 4.1. Let $\beta_{p}=\left.\operatorname{Ad} W_{p}\right|_{\mathscr{M}^{\alpha}}, p \in \widehat{G}$. Then $(\beta, \mathbf{I})$ is a predual cocycle crossed action and $(\beta, \mathbf{I})$ is cocycle conjugate to $(\check{\alpha}, U)$ by Proposition 3.4 and Lemma 3.7. We can then let $V$ be the unitary map from the definition of cocycle conjugacy. Conversely, assume $V$ exists. By construction of $(\check{\alpha}, U)$, there is a unitary map $S: \widehat{G} \rightarrow \mathscr{U}(\mathscr{M})$ with $S_{p} \in \mathscr{M}(p), p \in \widehat{G}, S_{0}=\mathbf{I}$, such that $\check{\alpha}_{p}=\operatorname{Ad} S_{p}$ and $U_{p, q}=S_{p} S_{q} S_{p+q}^{*}, p, q \in \widehat{G}$. Define $W_{p}=V_{p} S_{p}, p \in \widehat{G}$. Then $W$ satisfies the condition of Definition 4.1.

The condition of Lemma 4.2 can be stated shortly by saying that $U$ is an $\check{\alpha}$-coboundary, see [11, Definition 2.1.2].

Theorem 4.3. Let $G$ be second countable and let $\alpha: G \rightarrow$ Aut $\mathscr{M}$ be a prime action. If either

(i) $\mathscr{M}^{\alpha}$ is properly infinite, or

(ii) $G$ is finite and $\operatorname{dim} \mathscr{M}=\infty$,

then $\alpha$ is a dual action. 
Proof. Case (i) follows from Theorem 2.3 and [11, Theorem 4.3.3]. To prove case (ii), observe that $\alpha$ has full unitary spectrum by Proposition 2.7. Hence we can obtain the conclusion in the same way as above if we can show that $\mathscr{M}^{\alpha}$ is either properly infinite or type $\mathrm{II}_{1}$, cf. [11, Theorem 4.3.3]. However, $\operatorname{dim} \mathscr{M}^{\alpha}<\infty$ is excluded because we assume $|G|<\infty$ and $\operatorname{dim} \mathscr{M}=\infty$. The conclusion follows.

The case of ergodic actions studied in [9] shows that not all prime actions of $G$ are dual actions. However, it will follow from our main results that every prime action of a second countable $G$ on a semifinite injective von Neumann algebra with separable predual is a dual action provided the fixed point algebra is not finite dimensional. In view of this and the preceding theorem, one may wonder if all prime actions of $G$ with full unitary spectrum and infinite dimensional fixed point algebra are dual actions. As Lemma 4.2 demonstrates this is actually a problem in noncommutative cohomology.

Here we continue the investigation of the connection between $\mathscr{M}$ and $\mathscr{M}^{\alpha}$.

Lemma 4.4. Let $\alpha: G \rightarrow$ Aut $\mathscr{M}$ be a prime action with full unitary spectrum. If $\varphi$ is a normal trace state on $\mathscr{M}^{\alpha}$, the state $\varphi\left(\int_{G} \alpha_{g}(\cdot) d g\right)$ is a normal trace state on $\mathscr{M}$. In particular, $\mathscr{M}$ is finite if $\mathscr{M}^{\alpha}$ is.

Proof. For each $p \in \widehat{G}$, choose a unitary $U_{p} \in \mathscr{M}(p)$ and take $U_{0}=\mathbf{I}$. Set $\tilde{\varphi}(\cdot)=\varphi\left(\int_{G} \alpha_{g}(\cdot) d g\right)$. Since $\operatorname{span}\left\{a U_{p} \mid a \in \mathscr{M}^{\alpha}, p \in \widehat{G}\right\}$ is weakly dense in $\mathscr{M}$, it suffices to check that

$$
\tilde{\varphi}\left(a U_{p} b U_{q}\right)=\tilde{\varphi}\left(b U_{q} a U_{p}\right), \quad a, b \in \mathscr{M}^{\alpha}, p, q \in \widehat{G} .
$$

This is left to the reader.

Proposition 4.5. Assume that $G$ is second countable and let $\alpha: G \rightarrow$ Aut $\mathscr{M}$ be a prime action.

Then $\mathscr{M}$ is injective if and only if $\mathscr{M}^{\alpha}$ is injective. If $\mathscr{M}$ is not of type III, we have

(a) $\mathscr{M}$ is finite if and only if $\mathscr{M}^{\alpha}$ is finite,

(b) $\mathscr{M}$ is semifinite if and only if $\mathscr{M}^{\alpha}$ is semifinite.

Proof. If $\mathscr{M}$ is injective so is $\mathscr{M}^{\alpha}$ since $\int_{G} \alpha_{g}(\cdot) d g$ is a norm one projection onto $\mathscr{M}^{\alpha}$. Assume $\mathscr{M}^{\alpha}$ is injective. By Theorem 4.3, $\alpha \otimes$ id on $\mathscr{M} \otimes \mathscr{B}\left(l^{2}\right)$ is a dual action. Since $\mathscr{M} \otimes \mathscr{B}\left(l^{2}\right)^{\alpha \otimes \text { id }}=\mathscr{M}^{\alpha} \otimes \mathscr{B}\left(l^{2}\right)$ it follows from [2] that $\mathscr{M} \otimes \mathscr{B}\left(l^{2}\right)$ is injective. Hence $\mathscr{M}$ is injective.

(a) follows immediately from Theorem 2.3 and Lemma 4.4. To prove (b) observe that $\mathscr{M}^{\alpha}$ cannot be of type III when $\mathscr{M}$ is semifinite. This follows from [14, Chapter V, Lemma 2.29]. Assume finally that $\mathscr{M}^{\alpha}$ is semifinite. If $\mathscr{M}^{\alpha}$ is of type I, $\mathscr{M}=\left(\mathscr{M}^{\alpha}\right)^{\prime} \cap \mathscr{M} \otimes \mathscr{M}^{\alpha}$ and $\left.\alpha\right|_{\mathscr{M} \cap\left(\mathscr{M}^{\alpha}\right)^{\prime}}$ is ergodic. It follows from [9] that $\mathscr{M} \cap\left(\mathscr{M}^{\alpha}\right)^{\prime}$ is of type I or II. Hence $\mathscr{M}$ is semifinite in this case. 
If $\mathscr{M}^{\alpha}$ is of type $\mathrm{II}_{\infty}, \mathscr{M}^{\alpha}=\mathscr{N} \otimes \mathscr{B}$, where $\mathscr{N}$ is a finite factor and $\mathscr{B}$ is a type $\mathrm{I}_{\infty}$ factor, see [14, Chapter V, Proposition 1.40]. Then $\mathscr{M}=\mathscr{M}_{1} \otimes \mathscr{B}$, where $\mathscr{M}_{1}=\mathscr{M} \cap \mathscr{B}^{\prime}$. Since $\mathscr{M}$ is not of type III, $\mathscr{M}_{1}$ is not of type III either. As $\mathscr{N}$ is the fixed point algebra of $\left.\alpha\right|_{\mathscr{M}_{1}}$, we conclude from (a) that $\mathscr{M}_{1}$ is finite. Hence $\mathscr{M}$ is semifinite in this case also.

The results obtained in this section make it quite easy to show that the invariant $(\Omega, N)$ introduced in $\S 3$ for a prime $G$-action $\alpha$ with full unitary spectrum is the same as the characteristic invariant $\chi_{\hat{\alpha}}$ for the dual action $\hat{\alpha}$, see [5], in particular Remark 2.1.2. Since this observation will not be needed later, we only sketch the argument. Consider the action $\alpha \otimes$ id on $\mathscr{M} \otimes \mathscr{B}\left(l^{2}\right)$. Then $N(\alpha)=N(\alpha \otimes$ id $)$ and $\Omega(\alpha)=\Omega(\alpha \otimes$ id $)$. By Theorem $4.3 \alpha \otimes$ id is a dual action, i.e. there is an action $\beta$ of $\widehat{G}$ on $\mathscr{M}^{\alpha} \otimes \mathscr{B}\left(l^{2}\right)$ such that $\alpha \otimes$ id is conjugate to $\hat{\beta}$. Thus $N(\alpha)=N(\beta)$ and $\Omega(\alpha \otimes$ id $)=\Omega((\beta, \mathbf{I}))$ by Lemma 3.8. By the definitions of $\Omega((\beta, I))$ and $\chi_{\beta}$, these invariants agree. Using Takesaki duality and a result of Kallman [6], it follows easily that $\beta$ and the double dual action have the same invariant. Since $\hat{\hat{\beta}}$ is cocycle conjugate to the dual of $\alpha \otimes \mathrm{id}$, which is $\hat{\alpha} \otimes \mathrm{id}$, it suffices to observe that $\chi_{\hat{\alpha} \otimes i \mathrm{~d}}=\chi_{\hat{\alpha}}$.

\section{Actions with $\left(\mathscr{M}^{\alpha}\right)^{\prime} \cap \mathscr{M}=\mathbf{C}$}

Ocneanu has shown that each countable discrete amenable group has, up to cocycle conjugacy, precisely one free action on the hyperfinite $\mathrm{II}_{1}$-factor $\mathbf{R}$. He uses a cohomology vanishing result which, as we shall see, provides a direct way to translate his uniqueness result for abelian groups to a uniqueness result for actions by compact second countable abelian groups.

We apply the following lemma. It is well known for actions and the proof for cocycle crossed actions is the same. Therefore we omit it.

Lemma 5.1. Let $H$ be a discrete abelian group and $(\alpha, U)$ a cocycle crossed action on $\mathscr{M}$. Then each $\alpha_{h}, h \neq 0$, is free if and only if

$$
\mathscr{M}^{\prime} \cap \mathscr{M} \times_{\alpha, U} H \subseteq \mathscr{M} \text {. }
$$

Theorem 5.2. Let $\mathscr{M}$ be either the hyperfinite $\mathrm{II}_{1}-$ or $\mathrm{II}_{\infty}$-factor. Then every compact abelian second countable group has, up to conjugacy, precisely one action $\alpha$ on $\mathscr{M}$ with $\left(\mathscr{M}^{\alpha}\right)^{\prime} \cap \mathscr{M}=\mathbf{C}$.

Proof. The existence of such an action on $R$ follows by taking Ocneanu's free action and then let $\alpha$ be the dual action. An action on $R_{0,1}=R \otimes \mathscr{B}\left(l^{2}\right)$ is then obtained by taking $\alpha \otimes$ id. To prove uniqueness assume first that $\mathscr{M}=R_{0,1}$. By Proposition 4.5, $\mathscr{M}^{\alpha} \simeq \mathscr{B}\left(l^{2}\right)$ or $\mathscr{M}^{\alpha} \simeq R_{0,1}$. The first case is excluded because $\left(\mathscr{M}^{\alpha}\right)^{\prime} \cap \mathscr{M}=\mathbf{C}$, since $\mathscr{M}=\mathscr{M}^{\alpha} \otimes\left(\mathscr{M}^{\alpha}\right)^{\prime} \cap \mathscr{M}$ when $\mathscr{M}^{\alpha} \simeq \mathscr{B}\left(l^{2}\right)$. Thus $\mathscr{M}^{\alpha} \simeq R_{0,1}$, i.e. $\mathscr{M}^{\alpha}=\mathscr{N} \otimes \mathscr{B}$ where $\mathscr{B} \simeq \mathscr{B}\left(l^{2}\right)$ and $\mathscr{N} \simeq R$. It follows that $\mathscr{M}=\mathscr{B}^{\prime} \cap \mathscr{M} \otimes \mathscr{B}$ and that $\alpha=\left.\alpha\right|_{\mathscr{B} \prime \cap \mathscr{M}} \otimes$ id under this decomposition. The

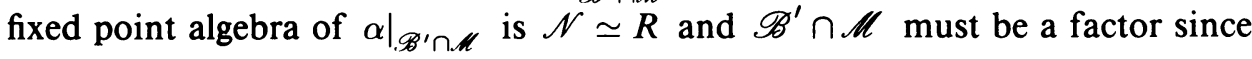


is. We therefore conclude from Proposition 4.5 that $\mathscr{B}^{\prime} \cap \mathscr{M} \simeq R$. Since $\mathbf{C}=\left(\mathscr{M}^{\alpha}\right)^{\prime} \cap \mathscr{M}=(\mathscr{N} \otimes \mathscr{B})^{\prime} \cap\left(\mathscr{B}^{\prime} \cap \mathscr{M}\right) \otimes \mathscr{B}=\mathscr{N}^{\prime} \otimes \mathscr{B}^{\prime} \cap\left(\mathscr{B}^{\prime} \cap \mathscr{M}\right) \otimes \mathscr{B}=$ $\mathscr{N}^{\prime} \cap \mathscr{B}^{\prime} \cap \mathscr{M} \otimes \mathbf{C}$, we have $\mathscr{N}^{\prime} \cap \mathscr{B}^{\prime} \cap \mathscr{M}=\mathbf{C}$. This reduces the proof to the case $\mathscr{M}=R$. In this case $\mathscr{M}^{\alpha}$ is finite so either $\operatorname{dim} \mathscr{M}^{\alpha}<\infty$ or $\mathscr{M}^{\alpha} \simeq R$. The first case is excluded by the same reasoning used above. Hence $\mathscr{M}^{\alpha} \simeq R$. By Lemma 5.1 any of $\alpha$ 's predual cocycle crossed actions on $\mathscr{M}^{\alpha}$ are free. Hence Ocneanu's cohomology vanishing theorem, [8, Theorem 7.6], combined with Lemma 4.2 yield that $\alpha$ is a dual action. Therefore the uniqueness of $\alpha$, up to conjugacy, follows from Ocneanu's uniqueness result for free actions by use of Lemma 3.7.

\section{THE MAIN RESULTS}

To obtain our main results we first have to investigate the case where the characteristic invariant is constant. We need the notion of induced actions, [12]. Let $G_{0} \subseteq G$ be a closed subgroup and let $\beta: G_{0} \rightarrow$ Aut $\mathscr{N}$ be an action. The sub von Neumann algebra of $L^{\infty}(G, \mathscr{N})$ consisting of the functions $y$ satisfying

$$
y(g-h)=\beta_{h}(y(g)), \quad g \in G, h \in G_{0},
$$

is denoted by $\operatorname{Ind}(\beta, \mathscr{N})$. The induced action $\tilde{\beta}$ of $G$ on $\operatorname{Ind}(\beta, \mathscr{N})$ is defined by

$$
\tilde{\beta}_{g}(y)(s)=y(s-g), \quad g, s \in G, y \in \operatorname{Ind}(\beta, \mathscr{N}) .
$$

In the following proof we follow the ideas used in [9] to prove Lemma 5.11 and Theorem 5.12.

Proposition 6.1. Let $\alpha: G \rightarrow$ Aut $\mathscr{M}$ be a prime action. Assume that $G$ is second countable, that $\mathscr{M}$ is finite and that $\Omega(\alpha)(p, q)=1$ for all $(p, q) \in \widehat{G} \times N(\alpha)$. Then there is a finite factor $\mathcal{N}$ and an action $\beta: N(\alpha)^{\perp} \rightarrow$ Aut $\mathscr{N}$ such that $\left(\mathscr{N}^{\beta}\right)^{\prime} \cap \mathscr{N}=\mathbf{C}$ and $\alpha$ is conjugate to $\tilde{\boldsymbol{\beta}}$.

Proof. Choose unitaries $V_{p} \in \mathscr{M}(p), p \in \widehat{G}$, such that $V_{q} \in \mathscr{M}(q) \cap\left(\mathscr{M}^{\alpha}\right)^{\prime}$, $q \in N(\alpha)$. By assumption $V_{p} V_{q}=V_{q} V_{p}, p \in \widehat{G}, q \in N(\alpha)$. It follows that $\mathscr{M} \cap\left(\mathscr{M}^{\alpha}\right)^{\prime}=Z_{\mathscr{M}}$, the center of $\mathscr{M}$. Since $\mathscr{M}$ is finite we have a unique centervalued trace $T: \mathscr{M} \rightarrow Z_{\mathscr{M}}$. Set

$$
\mathscr{M}_{c}=\left\{m \in \mathscr{M} \mid \alpha_{g}(m) \text { is norm-continuous in } g\right\} .
$$

It is well known that $\mathscr{M}_{c}$ is a weakly dense $C^{*}$-subalgebra of $\mathscr{M}$. Since $\alpha_{g} \circ T=$ $T \circ \alpha_{g}, g \in G$, by the uniqueness of $T$, we see that $T$ leaves $\mathscr{M}_{c}$ globally invariant and $T\left(\mathscr{M}_{c}\right)=Z_{\mathscr{M}_{c}}$, the center of $\mathscr{M}_{c}$. Let $\omega$ be a pure state of $Z_{\mathscr{M}_{c}}$. Then $\tau_{0} \equiv \omega \circ T$ is a trace state on $\mathscr{M}_{c}$. Let $\left(\pi_{0}, \mathscr{H}_{0}, \psi_{0}\right)$ be the corresponding GNS-representation. When $t \in N(\alpha)^{\perp}, \alpha_{t}$ acts trivially on $Z_{\mathscr{M}}$ since $\alpha_{t}\left(V_{q}\right)=V_{q}$ for all $q \in N(\alpha)$. Therefore $T \circ \alpha_{t}=T$ by the uniqueness 
of $T$. It follows that $\tau_{0} \circ \alpha_{t}=\tau_{0}, t \in N(\alpha)^{\perp}$. We can then define unitaries $W_{t}$ on $\mathscr{H}_{0}$ by

$$
W_{t} \pi_{0}(x) \psi_{0}=\pi_{0}\left(\alpha_{t}(x)\right) \psi_{0}, \quad t \in N(\alpha)^{\perp}, x \in \mathscr{M}_{c} .
$$

Then $\left(\pi_{0}, W\right)$ is a covariant representation of $\left(\mathscr{M}_{c},\left.\alpha\right|_{N(\alpha)^{\perp}}\right)$. Let $\mathscr{N}=\pi_{0}\left(\mathscr{M}_{c}\right)^{\prime \prime}$ and define $\beta_{t} \in$ Aut $\mathscr{N}$ by $\beta_{t}=\operatorname{Ad} W_{t}, t \in N(\alpha)^{\perp}$. Define $\Phi: \mathscr{M}_{c} \rightarrow$ $L^{\infty}(G, \mathcal{N})$ by

$$
\Phi(x)(g)=\pi_{0}\left(\alpha_{-g}(x)\right), \quad x \in \mathscr{M}_{c}, g \in G .
$$

Then

$$
\begin{aligned}
\Phi(x)(g-t) & =\pi_{0}\left(\alpha_{-g+t}(x)\right)=\pi_{0}\left(\alpha_{t}(x)\right) \\
& =\beta_{t}\left(\pi_{0}\left(\alpha_{-g}(x)\right)\right), \quad g \in G, t \in N(\alpha)^{\perp} .
\end{aligned}
$$

Thus $\Phi\left(\mathscr{M}_{c}\right) \subseteq \operatorname{Ind}(\beta, \mathscr{N})$.

Next we identify $\Phi\left(Z_{\mathscr{M}_{c}}\right)$. For this we let $G \ni s \rightarrow \dot{s} \in G / N(\alpha)^{\perp}$ denote the quotient map and let $\dot{\alpha}: G / N(\alpha)^{\perp} \rightarrow \operatorname{Aut}\left(Z_{\mathscr{M}_{c}}\right)$ be the action $\dot{\alpha}_{\dot{s}}=\alpha_{s}, s \in G$. This action is ergodic, so it is conjugate to left translation on $C\left(G / N(\alpha)^{\perp}\right)$ by [1], Theorem 2.2. Identifying $Z_{\mathscr{M}_{c}}$ with $C\left(G / N(\alpha)^{\perp}\right)$ in this way we see that

$$
\Phi(f)(g)=f(\dot{r}-\dot{g}), \quad g \in G, \text { for some point } r \in G
$$

and all $f \in Z_{\mathscr{M}_{c}}$. This follows because $\omega$ is pure so that $\pi_{0}$ is a onedimensional representation when restricted to $Z_{\mathscr{M}_{c}}$. It follows that $\Phi$ maps $Z_{\mathscr{M}_{c}}$ onto the $C^{*}$-subalgebra of $C(G) \subseteq L^{\infty}(G, \mathscr{N})$ consisting of functions $f$ satisfying $f(g)=f(g+t), g \in G, t \in N(\alpha)^{\perp}$. Now the partition of unity argument from Olesen, Pedersen and Takesaki's proof of Lemma 5.11 in [9] applies to conclude that $\Phi$ maps $\mathscr{M}_{c}$ onto the elements $y$ in $C\left(G, \pi_{0}\left(\mathscr{M}_{c}\right)\right) \subseteq L^{\infty}(G, \mathscr{N})$ satisfying

$$
y(g-t)=\beta_{t}(y(g)), \quad g \in G, t \in N(\alpha)^{\perp} .
$$

Consider $L^{\infty}(G, \mathscr{N})$ represented on $L^{2}\left(G, \mathscr{H}_{0}\right)$ in the obvious way. $\operatorname{Ind}(\beta, \mathscr{N})$ is the fixed point algebra of an action of $N(\alpha)^{\perp}$ on $L^{\infty}(G, \mathcal{N})$, see [12, p. 299]. Using the corresponding normal conditional expectation and the preceding description of $\Phi\left(\mathscr{M}_{c}\right)$, one concludes easily from the weak density of $C\left(G, \pi_{0}\left(\mathscr{M}_{c}\right)\right)$ in $L^{\infty}(G, \mathscr{N})$ that the weak closure of $\Phi\left(\mathscr{M}_{c}\right)$ in $\mathscr{B}\left(L^{2}\left(G, \mathscr{H}_{0}\right)\right)$ is $\operatorname{Ind}(\beta, \mathscr{N})$. We argue that $\Phi$ extends to a *-isomorphism from $\mathscr{M}$ onto $\operatorname{Ind}(\beta, \mathscr{N})$. To this end consider $\psi=\mathbf{I} \otimes \psi_{0}$ in $L^{2}\left(G, \mathscr{H}_{0}\right)$. We have

$$
(\Phi(x) \psi, \psi)=\int_{G} \tau_{0}\left(\alpha_{-g}(x)\right) d g, \quad x \in \mathscr{M}_{c} .
$$

Thus $(\Phi(\cdot) \psi, \psi)$ is an $\alpha$-invariant trace state on $\mathscr{M}_{c}$. But $\mathscr{M}^{\alpha} \subseteq \mathscr{M}_{c}$ is a finite factor with a unique trace $\varphi$, so there is only one $\alpha$-invariant trace state on $\mathscr{M}_{c}$, viz. $\varphi\left(\int_{G} \alpha_{g}(\cdot) d g\right)$. Let $\mathscr{H}=\overline{\Phi\left(\mathscr{M}_{c}\right) \psi}$. Then $(\Phi, \mathscr{H}, \psi)$ is a cyclic 
representation and the uniqueness of the GNS-construction together with the faithfulness and normality of $\varphi\left(\int_{G} \alpha_{g}(\cdot) d g\right)$ on $\mathscr{M}$ imply that $\Phi$ extends to a $*$-isomorphism mapping $\mathscr{M}$ onto the weak closure of $\Phi\left(\mathscr{M}_{c}\right)$ in $\mathscr{B}(\mathscr{H})$. To prove our assertion it is therefore sufficient to show that reduction to $\mathscr{H}$ is a *-isomorphism on $\operatorname{Ind}(\beta, \mathscr{N})$. But this follows from the equality

$$
(y \psi, \psi)=\int_{G}\left(y(g) \psi_{0}, \psi_{0}\right) d g, \quad y \in \operatorname{Ind}(\beta, \mathscr{N}),
$$

and the fact that $\psi_{0}$ is separating for $\mathcal{N}$ as a cyclic trace vector. Thus $\Phi$ extends to a $*$-isomorphism of $\mathscr{M}$ onto $\operatorname{Ind}(\beta, \mathscr{N})$ as asserted. Note that

$$
\begin{aligned}
\Phi\left(\alpha_{g}(x)\right)(s) & =\pi_{0}\left(\alpha_{g-s}(x)\right)=\Phi(x)(s-g) \\
& =\tilde{\beta}_{g}(\Phi(x))(s), \quad g, s \in G, x \in \mathscr{M}_{c},
\end{aligned}
$$

so that the extension of $\Phi$ conjugates $\alpha$ and $\tilde{\beta}$.

To complete the proof it suffices to check that $\left(\mathscr{N}^{\beta}\right)^{\prime} \cap \mathcal{N}=\mathbf{C}$ since $\mathscr{N}$ is then necessarily a factor and finite since $\psi_{0}$ is a trace vector. Since $G$ is second countable there is a Borel right inverse $q$ for the quotient map $G \rightarrow G / N(\alpha)^{\perp}$. If $b \in\left(\mathscr{N}^{\beta}\right)^{\prime} \cap \mathcal{N}$, we define $Y_{b} \in \operatorname{Ind}(\beta, \mathscr{N})$ by

$$
Y_{b}(g)=\beta_{q(\dot{g})-g}(b), \quad g \in G .
$$

We argue that $Y_{b}$ is in the commutant of $\operatorname{Ind}(\beta, \mathscr{N})^{\tilde{\beta}}$. But it is easy to see that elements in $\operatorname{Ind}(\beta, \mathscr{N})^{\tilde{\beta}}$ are represented by functions on $G$ taking a constant value in $\mathscr{N}^{\beta}$. Since $b \in\left(\mathscr{N}^{\beta}\right)^{\prime} \cap \mathcal{N}$, it follows that $Y_{b}$ commutes with elements in $\operatorname{Ind}(\beta, \mathscr{N})^{\tilde{\beta}}$. Thus $\Phi^{-1}\left(Y_{b}\right) \in\left(\mathscr{M}^{\alpha}\right)^{\prime} \cap \mathscr{M}=Z_{\mathscr{M}}$. But we saw above that $\Phi\left(Z_{\mathscr{M}_{c}}\right) \subseteq C(G)$, so the weak density of $Z_{\mathscr{M}_{c}}$ in $Z_{\mathscr{M}}$ implies that $\Phi\left(Z_{\mathscr{M}}\right) \subseteq$ $L^{\infty}(G) \subseteq L^{\infty}(G, \mathscr{N})$. Thus $Y_{b} \in L^{\infty}(G)$, i.e. $b \in \mathbf{C}$.

We can now extend a result of Ocneanu, [8, Theorem 2.6], to abelian cocycle crossed actions.

Theorem 6.2. Let $H$ be a countable discrete abelian group and let $(\alpha, U)$ and $(\beta, V)$ be two cocycle crossed actions of $H$ on the hyperfinite II $_{1}$-factor $R$. Then $(\alpha, U)$ and $(\beta, V)$ are cocycle conjugate if and only of $N(\alpha)=N(\beta)$ and $\Omega(\alpha, U)=\Omega(\beta, V)$.

Proof. The necessity of the two conditions follows from $\S 3$. To prove sufficiency fix the subgroup $N=N(\alpha)=N(\beta)$. Let $\mathscr{S}$ denote the equivalence classes of cocycle crossed actions $(\alpha, U)$ of $H$ on $R$ with $N(\alpha)=N$. For such an action we let $[(\alpha, U)]$ denote the corresponding element in $\mathscr{S}$. We can then use that $R \otimes R \simeq R$ and a result of Kallman, [6], to define a semigroup structure on $\mathscr{S}$ by

$$
[(\alpha, U)][(\beta, V)]=[(\alpha \otimes \beta, U \otimes V)] .
$$

Let $L$ denote the group of bihomomorphisms from $\widehat{G} \times N$ into $\mathbf{T}$ which are antisymmetric on $N \times N$. Then $[(\alpha, U)] \rightarrow \Omega(\alpha, U) \in L$ is a well-defined 
semigroup homomorphism. By [3, Theorem 2.1], there is for each $\lambda \in L$ an action $j^{\lambda}: H \rightarrow$ Aut $R$ such that $N\left(j^{\lambda}\right)=N$ and $\Omega\left(\left(j^{\lambda}, \mathbf{I}\right)\right)=\lambda$. Define $\lambda[(\alpha, U)]=\left[\left(j^{\lambda} \otimes \alpha, \mathbf{I} \otimes U\right)\right]$. Since $j^{\lambda_{1}} \otimes j^{\lambda_{2}}$ and $j^{\lambda_{1} \lambda_{2}}$ are cocycle conjugate by [8, Theorem 2.6], this definition makes $\mathscr{S}$ into a left $L$ space and the homomorphism $\mathscr{S} \rightarrow L$ into a homomorphism of $L$-spaces. By [8, Lemma $2.5(\mathrm{~b})]$ it therefore suffices to show that there is only one element in $\mathscr{S}$ mapping onto the identity of $L$. But this follows by combining Lemma 3.7, Theorem 5.2 and Proposition 6.1.

Corollary 6.3. Let $(\alpha, U)$ be a cocycle crossed action of $H$ on $R$. Then $U$ is an $\alpha$-coboundary, i.e. there is a unitary map $V: H \rightarrow R$ such that $U_{g, h}=$ $\alpha_{g}\left(V_{h}^{*}\right) V_{g}^{*} V_{g+h}, g, h \in H$.

Proof. Combining Theorem 6.2 with Theorem 2.1 of [3] one sees that $(\alpha, U)$ is cocycle conjugate to $(\beta, \mathbf{I})$, where $\beta$ is an action. The conclusion then follows from condition (a) of Definition 3.6.

Theorem 6.4. Let $\mathscr{M}$ be a semifinite, injective von Neumann algebra with separable predual and let $\alpha, \beta: G \rightarrow$ Aut $\mathscr{M}$ be two prime actions of the second countable compact abelian group $G$. Then $\alpha$ and $\beta$ are conjugate if and only if $N(\alpha)=N(\beta), \Omega(\alpha)=\Omega(\beta)$ and $\mathscr{M}^{\alpha} \simeq \mathscr{M}^{\beta}$.

Proof. The necessity of the conditions follows from $\S 3$. To prove sufficiency observe that there are 3 possibilities for $\mathscr{M}^{\alpha}$ :

(i) $\mathscr{M}^{\alpha} \simeq \mathscr{B}(\mathscr{H})$ for some separable Hilbert space $\mathscr{H}$,

(ii) $\mathscr{M}^{\alpha} \simeq R$,

(iii) $\mathscr{M}^{\alpha} \simeq R_{0,1}$.

In case (i) $\mathscr{M}=\mathscr{M}^{\alpha} \otimes\left(\mathscr{M}^{\alpha}\right)^{\prime} \cap \mathscr{M}$ and $\left.\alpha\right|_{\left(\mathscr{M}^{\alpha}\right)^{\prime} \cap \mathscr{M}}$ is ergodic. Since $\alpha$ is faithful, so is $\left.\alpha\right|_{(\mathscr{M})^{\prime} \cap \mathscr{M}}$. Whence $N(\alpha)=\widehat{G}$ and by the same reasoning $N(\beta)=\widehat{G}$. In this case $\Omega(\alpha)$ and $\Omega(\beta)$ are the invariants of Olesen, Pedersen and Takesaki [9]. So in this case $\alpha$ and $\beta$ must be conjugate because of [9], Theorem 4.5.

Next suppose $\mathscr{M}^{\alpha} \simeq \mathscr{M}^{\beta} \simeq R_{0,1}=R \otimes \mathscr{B}(\mathscr{H})$. Let $\mathscr{B}_{1}, \mathscr{B}_{2}$ be $\mathrm{I}_{\infty}$ subfactors of $\mathscr{M}$ such that $\mathscr{M}^{\alpha}=\mathscr{N}_{1} \otimes \mathscr{B}_{1}$ and $\mathscr{M}^{\beta}=\mathscr{N}_{2} \otimes \mathscr{B}_{2}$, where $\mathscr{N}_{1}=\mathscr{M}^{\alpha} \cap \mathscr{B}_{1}^{\prime} \simeq R$ and $\mathscr{N}_{2}=\mathscr{M}^{\beta} \cap \mathscr{B}_{2}^{\prime} \simeq R$. Then $\mathscr{M}=\mathscr{M}_{1} \otimes \mathscr{B}_{1}=\mathscr{M}_{2} \otimes \mathscr{B}_{2}$, where $\mathscr{M}_{i}=\mathscr{M} \cap \mathscr{B}_{i}^{\prime}, i=1,2$. It follows that $\alpha=\alpha_{1} \otimes$ id, $\beta=\beta_{1} \otimes$ id, where $\alpha_{1}=\left.\alpha\right|_{\mathscr{M}_{1}}$ and $\beta_{1}=\left.\beta\right|_{\mathscr{M}_{2}}$. Then $N\left(\alpha_{1}\right)=N\left(\beta_{1}\right), \Omega\left(\alpha_{1}\right)=\Omega\left(\beta_{1}\right)$ and $\mathscr{M}_{1}^{\alpha_{1}}=\mathscr{N}_{1} \simeq R \simeq \mathscr{N}_{2}=\mathscr{M}_{2}^{\beta_{1}}$. Therefore it suffices to consider case (ii). But in this case we can apply Proposition 3.4, Lemma 3.8, Theorem 6.2 and finally Lemma 3.7 to conclude that $\alpha$ and $\beta$ are conjugate.

\section{Almost PERIOdic aCtions of locally COMPACT ABELIAN GROUPS}

Now let $\Gamma$ be a locally compact abelian group, and consider a faithful action $\alpha: \Gamma \rightarrow$ Aut $\mathscr{M}$. As before $\mathscr{M}(p)$ denotes the eigenspace corresponding to the 
continuous character $p \in \widehat{\Gamma}$ and define the discrete spectrum $\operatorname{Sp}_{d}(\alpha)$ of $\alpha$ to be

$$
\operatorname{Sp}_{d}(\alpha)=\{p \in \widehat{\Gamma} \mid \mathscr{M}(p) \neq 0\}
$$

In the following definition we follow [9].

Definition 7.1. $\alpha$ is called almost periodic if the linear map of the subspaces $\mathscr{M}(p), p \in \widehat{\Gamma}$, is weakly dense in $\mathscr{M}$ and there is a faithful normal $\alpha$-invariant state on $\mathscr{M}$.

It is well known that the existence of a faithful normal state requires $\mathscr{M}$ to be $\sigma$-finite. So almost periodic actions are always acting on $\sigma$-finite von Neumann algebras.

Example 7.2. Let $H \subseteq \widehat{\Gamma}$ be a dense subgroup and consider $H$ as a discrete group. Let $\beta: H \rightarrow$ Aut $\mathscr{M}$ be an action on a $\sigma$-finite von Neumann algebra $\mathscr{M}$. Let $\hat{\beta}: \widehat{H} \rightarrow$ Aut $\mathscr{M} \times_{\beta} H$ be the dual action and $i: \Gamma \rightarrow \widehat{H}$ the obvious continuous dense embedding. Define $\alpha_{g}=\hat{\beta}_{i(g)}, g \in \Gamma$. Then $\alpha$ is almost periodic.

Proposition 7.3. Assume $\alpha$ is a prime action. Then $\alpha$ is almost periodic if and only if $\operatorname{Sp}_{d}(\alpha)$ is a dense (discrete) subgroup of $\widehat{\Gamma}$ and there is an action $\beta: \operatorname{Sp}_{d}^{\wedge}(\alpha) \rightarrow$ Aut $\mathscr{M}$ such that $\alpha_{g}=\beta_{i(g)}, g \in \widehat{\Gamma}$, where $i: \Gamma \rightarrow \operatorname{Sp}_{d}^{\wedge}(\alpha)$ is the obvious continuous dense embedding.

Proof. The sufficiency of the conditions are clear, so we assume that $\alpha$ is almost periodic. Let $H$ be the subgroup of $\widehat{\Gamma}$ generated by $\operatorname{Sp}_{d}(\alpha)$. By assumption $\bigcup_{q \in H} \mathscr{M}(q)$ spans a weakly dense subspace $\mathscr{M}_{0}$ of $\mathscr{M}$. Since $H$ is a group, $\mathscr{M}_{0}$ is a *-subalgebra. We argue that each element $m$ of $\mathscr{M}_{0}$ admits a unique decomposition $m=\sum_{q \in H} a_{q}$, where $a_{q} \in \mathscr{M}(q), q \in H$. To see this let $\omega$ be a faithful normal $\alpha$-invariant state. Then $\omega(\mathscr{M}(p))=0$ for $p \in \widehat{\Gamma} \backslash\{0\}$ because we have

$$
\omega(a)=\omega\left(\alpha_{g}(a)\right)=\langle p, g\rangle \omega(a), \quad a \in \mathscr{M}(p), g \in \Gamma .
$$

If $\sum_{q \in H} a_{q}=0, a_{q} \in \mathscr{M}(q)$, we have

$$
\omega\left(a_{q_{0}} a_{q_{0}}^{*}\right)=\omega\left(\sum_{q \in H} a_{q} a_{q_{0}}^{*}\right)=0, \quad q_{0} \in H .
$$

Since $\omega$ is faithful, $a_{q}=0$ for all $q \in H$, proving the assertion. Fix $j \in \widehat{H}$. We can define a bijective $*$-homomorphism $\beta_{j}: \mathscr{M}_{0} \rightarrow \mathscr{M}_{0}$ by

$$
\beta_{j}\left(\sum_{q \in H} a_{q}\right)=\sum_{q \in H}\langle q, j\rangle a_{q} .
$$

Then $\omega \circ \beta_{j}=\omega$ on $\mathscr{M}_{0}$, so by use of the GNS-representation constructed from $\omega$, one sees that $\beta_{j}$ extends to a $*$-automorphism of $\mathscr{M}$. In the same 
representation of $\mathscr{M}$ it is then easy to check that $j \rightarrow \beta_{j}$ defines a continuous action of $H$ on $\mathscr{M}$. Clearly, $\alpha_{g}=\beta_{i(g)}, g \in \Gamma$, so the proof is complete when we show that $H=\operatorname{Sp}_{d}(\alpha)$. This follows as in the proof of Lemma 2.2.

Now let $\alpha: \Gamma \rightarrow$ Aut $\mathscr{M}$ be an almost periodic prime action. We define $N(\alpha)=\operatorname{Sp}_{d}\left(\left.\alpha\right|_{\left(\mathscr{M}^{\alpha}\right)^{\prime} \cap \mathscr{M}}\right)$. By Theorem 3.2 and Proposition 7.3, $\mathscr{M}(p)$ contains a unitary for all $p \in \operatorname{Sp}_{d}(\alpha)$, provided $\mathscr{M}$ is not of type III or $\mathscr{M}^{\alpha}$ not of type $\mathrm{II}_{1}$. We can then choose unitaries $U_{p} \in \mathscr{M}(p), p \in \mathrm{Sp}_{d}(\alpha)$, such that $U_{q} \in\left(\mathscr{M}^{\alpha}\right)^{\prime} \cap \mathscr{M}(q)$ when $q \in N(\alpha)$ and define $\Omega(\alpha): \operatorname{Sp}_{d}(\alpha) \times N(\alpha) \rightarrow \mathbf{T}$ by $\Omega(\alpha)(p, q)=U_{p} U_{q} U_{p}^{*} U_{q}^{*}$. Combination of Proposition 7.3 with Theorem 6.4 yields the following result.

Theorem 7.4. Let $\alpha, \beta: \Gamma \rightarrow$ Aut $\mathscr{M}$ be two faithful almost periodic prime actions on the injective semifinite von Neumann algebra $\mathscr{M}$. Then $\alpha$ and $\beta$ are conjugate if and only if $\mathscr{M}^{\alpha} \simeq \mathscr{M}^{\beta}, \mathrm{Sp}_{d}(\alpha)=\mathrm{Sp}_{d}(\beta), N(\alpha)=N(\beta)$ and $\Omega(\alpha)=\Omega(\beta)$.

Let us finally mention the following result concerning the existence of almost periodic actions on $R$ and $R_{0,1}$. It follows readily from Theorem 5.2.

Theorem 7.5. Let $\mathscr{M}$ be either the hyperfinite $\mathrm{II}_{1}-$ or $\mathrm{II}_{\infty}$-factor. For each countable dense subgroup $H \subseteq \widehat{\Gamma}$, there is, up to conjugacy, precisely one almost periodic action $\alpha: \Gamma \rightarrow$ Aut $\mathscr{M}$ such that $\operatorname{Sp}_{d}(\alpha)=H$ and $\left(\mathscr{M}^{\alpha}\right)^{\prime} \cap \mathscr{M}=\mathbf{C}$.

\section{SUPPLEMENTARY RESUltS}

Let $\alpha: G \rightarrow$ Aut $\mathscr{M}$ be an action and assume that $\mathscr{M}^{\alpha}$ is a II $\infty_{\infty}^{- \text {factor. By }}$ Theorem $4.3 \alpha$ is a dual action, i.e. there is an action $\beta: \widehat{G} \rightarrow$ Aut $\mathscr{M}^{\alpha}$ such that $\alpha$ is conjugate to $\hat{\beta}$. Thus we can find a unitary representation $U: \widehat{G} \rightarrow \mathscr{U}(\mathscr{M})$ such that $\alpha_{g}\left(U_{p}\right)=\langle p, g\rangle U_{p}, g \in G$, and $\operatorname{Ad} U_{p \mid \mathscr{M}} \alpha=\beta_{p}, p \in \widehat{G}$. Choose a normal semifinite faithful trace $\tau$ on $\mathscr{M}^{\alpha}$ and define $\bmod (\alpha): \widehat{G} \rightarrow \mathbf{R}^{+}$by $\tau\left(U_{p} \cdot U_{p}^{*}\right)=\bmod (\alpha)(p) \tau(\cdot), p \in \widehat{G}$. Then $\bmod (\alpha)$ is a homomorphism. Combining Lemma 3.7 and Lemma 3.8 with [8, Theorem 2.9], we get the following result.

Theorem 8.1. Let $\alpha, \beta: G \rightarrow$ Aut $\mathscr{M}$ be two actions of the same compact abelian second countable group $G$ on the injective von Neumann $\mathscr{M}$ with separable predual. Assume that $\mathscr{M}^{\alpha}$ and $\mathscr{M}^{\beta}$ are $\mathrm{II}_{\infty}$-factors. Then $\alpha$ and $\beta$ are conjugate if and only if $N(\alpha)=N(\beta), \Omega(\alpha)=\Omega(\beta)$ and $\bmod (\alpha)=\bmod (\beta)$.

It should be noted that the algebra $\mathscr{M}$ in Theorem 8.1 may very well be of type III.

Notice that Theorem 8.1 admits a generalization to almost periodic actions along this lines of $\S 7$. 
If $\alpha$ is a prime $G$-action on a type III von Neumann algebra, then $\mathscr{M}^{\alpha}$ is either of type II or III. If $\mathscr{M}^{\alpha}$ is of type III, we know from Theorem 4.3 that $\alpha$ is a dual action. There does not seem to be much more we can say in this case. If $\mathscr{M}^{\alpha}$ is of type II and $G=\mathrm{T}$, we can say much more. In fact we get

Theorem 8.2. Let $\alpha: \mathbf{T} \rightarrow$ Aut $\mathscr{M}$ be an action on a type III von Neumann algebra. Assume that $\mathscr{M}^{\alpha}$ is a type II factor. Then there is a normal semifinite faithful weight $\varphi$ on $\mathscr{M}$ and a real number $a \in] 0,1[$ such that the modular automorphisms group $\sigma^{\varphi}$ satisfies

$$
\sigma_{t}^{\varphi}=\alpha_{a^{i t}}
$$

or

$$
\sigma_{-t}^{\varphi}=\alpha_{a^{i t}}, \quad t \in \mathbf{R} .
$$

Furthermore, $\mathscr{M}$ is a $\mathrm{III}_{\lambda}$-factor where $\lambda=e^{2 \pi / \log a}$ and $\left(\mathscr{M}^{\alpha}\right)^{\prime} \cap \mathscr{M}=\mathbf{C} . \mathscr{M}^{\alpha}$ is of type $\mathrm{II}_{1}$ if and only if $\varphi$ is finite.

Proof. Assume first that $\mathscr{M}^{\alpha}$ is a $\mathrm{II}_{1}$-factor. Using Lemma 2.4 and Lemma 4.4, we see that $\mathscr{M}$ is generated by $\mathscr{M}^{\alpha}$ and an isometry $V$, which is not a unitary and satisfies $\alpha_{\lambda}(V)=\lambda V$ or $\alpha_{\lambda}(V)=\bar{\lambda} V, \lambda \in \mathbf{T}$. Assume that the first alternative holds, and set $e=V V^{*} \in \mathscr{M}^{\alpha}$. Then $\operatorname{Ad} V: \mathscr{M}^{\alpha} \rightarrow e \mathscr{M}^{\alpha} e$ is a *-isomorphism and it follows by combining [13, Lemma 2.3], with Lemma 3.5 above, that $\mathscr{M} \simeq \mathscr{R}\left(\mathscr{M}^{\alpha}, \operatorname{Ad} V\right)$ in the notation of [13]. By [13, §2], there is a faithful normal state $\varphi$ on $\mathscr{M}$ such that $\sigma_{t}^{\varphi}(x)=x, x \in \mathscr{M}^{\alpha}$, and $\sigma_{t}^{\varphi}(V)=a^{i t} V, t \in \mathbf{R}$, where $\left.a=\varphi(e) \in\right] 0,1$ [. By [13, Corollary 2.15], $\varphi$ is a homogeneous state, so $\left(\mathscr{M}^{\alpha}\right)^{\prime} \cap \mathscr{M}=\mathbf{C}$ by [13, Proposition 1.7]. By

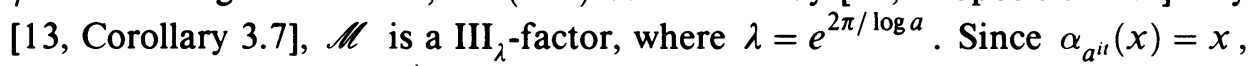
$x \in \mathscr{M}^{\alpha}$, and $\alpha_{a^{i t}}(V)=a^{i t} V$, we conclude that $\sigma_{t}^{\varphi}=\alpha_{a^{i t}}, t \in \mathbf{R}$. If the second alternative holds we can use the same reasoning on the action $\lambda \rightarrow \alpha_{\bar{\lambda}}, \lambda \in \mathbf{T}$.

Next assume that $\mathscr{M}^{\alpha}$ is a II ${ }_{\infty}$-factor. Then $\mathscr{M}^{\alpha}=\mathscr{N} \otimes \mathscr{B}$, where $\mathscr{N}$ is a $\mathrm{II}_{1}$-factor and $\mathscr{B}$ is a $\mathrm{I}_{\infty}$-factor. Then $\mathscr{M}=\mathscr{M}_{1} \otimes \mathscr{B}$ where $\mathscr{M}_{1}=\mathscr{B}^{\prime} \cap \mathscr{M}$ is of type III. By the first step in the proof we know that there is a faithful normal state $\varphi$ on $\mathscr{M}_{1}$ and $\left.a \in\right] 0,1\left[\right.$ such that $\sigma_{t}^{\varphi}=\alpha_{a^{i t}}$ or $\sigma_{-t}^{\varphi}=\alpha_{a^{i t}}$ on $\mathscr{M}_{1}$, that $\mathscr{M}_{1}$ is a $\mathrm{III}_{\lambda}$-factor, $\lambda=e^{2 \pi / \log a}$, and that $\mathscr{N}^{\prime} \cap \mathscr{M}_{1}=\mathbf{C}$. The conclusion for the $\mathrm{II}_{\infty}$-case follows by considering the weight $\varphi \otimes \operatorname{Tr}$ on $\mathscr{M}=\mathscr{M}_{1} \otimes \mathscr{B}$, where $\operatorname{Tr}$ is the trace on $\mathscr{B}$. Since

$$
\sigma^{\varphi \otimes \operatorname{Tr}}=\sigma^{\varphi} \otimes \mathrm{id},
$$

it is now obvious how to conclude the proof.

\section{REFERENCES}

1. S. Albeverio and R. Høegh-Krohn, Ergodic actions by compact groups on $C^{*}$-algebras, Math. Z. 174 (1980), 1-17.

2. A. Connes, Classification of injective factors, Ann. of Math. 104 (1976), 73-115. 
3. V. F. R. Jones, An invariant for group actions, Algèbres d'Opérateurs, Lecture Notes in Math., vol. 725, Springer-Verlag, New York, 1979, pp. 237-253.

4. __ Prime actions of compact abelian groups on the hyperfinite $\mathrm{II}_{1}$ factor, J. Operator Theory 9 (1983), 181-186.

5. V. F. R. Jones and M. Takesaki, Actions of compact abelian groups on semifinite injective factors, Acta Math. 153 (1984), 212-258.

6. R. Kallman, A decomposition theorem for automorphisms of von Neumann algebras, Functional Analysis (C. O. Wilde, ed.), Academic Press, New York, 1970, 33-35.

7. M. Landstad, Duality theory of covariant systems, Trans. Amer. Math. Soc. 248 (1979), 223267.

8. A. Ocneanu, Discrete amenable groups on von Neumann algebras, Lecture Notes in Math., vol. 1138, Springer-Verlag, New York, 1985.

9. D. Olesen, G. K. Pedersen and M. Takesảki, Ergodic actions of compact abelian groups, J. Operator Theory 3 (1980), 237-269.

10. W. Paschke, Inner product modules arising from compact automorphism groups on von Neumann algebras, Trans. Amer. Math. Soc. 224 (1976), 87-102.

11. C. Sutherland, Cohomology and extensions of operator algebras. II, Publ. RIMS Kyoto Univ. 16 (1980), 135-174.

12. M. Takesaki, Duality for crossed products and the structure of von Neumann algebras of type III, Acta Math. 131 (1973), 249-310.

13. _ The structure of a von Neumann algebra with a homogeneous periodic state, Acta Math. 131 (1973), 78-121.

14. __ Theory of operator algebras. I, Springer-Verlag, New York, 1979.

Matematisk Institut, Ny Munkegade, DK-8000 Aarhus C, Denmark 